\title{
Effects of temporal stimuli in the acquisition of a serial tracking task
}

\author{
Maria Teresa Cattuzzo' \\ Go Tani² \\ 'Physical Education Higher School, \\ University of Pernambuco, Recife, \\ Brazil; ${ }^{2}$ School of Physical Education \\ and Sports, University of São Paulo, \\ São Paulo, Brazil
}

This article was published in the following Dove Press journal:

Psychology Research and Behavior Management

18 July 2012

Number of times this article has been viewed

\begin{abstract}
This study investigated the effects of temporal stimuli on qualitative responses during the acquisition of a serial tracking task. One hundred and twenty young adult men performed 100 trials of a tracking task that consisted of touching six response keys in a given sequence in response to flashing light-emitting diodes in order to identify and learn the serial pattern. Six experimental groups were created with diverse inter stimuli intervals (ISI): G1: ISI $=300 \mathrm{~ms}$; G2: ISI $=400 \mathrm{~ms}$; G3: ISI $=500 \mathrm{~ms}$; G4: ISI $=600 \mathrm{~ms} ; \mathrm{G} 5:$ ISI $=700 \mathrm{~ms}$; and G6: ISI $=800 \mathrm{~ms}$. Performance was assessed by means of four types of responses: omission, error, correct, and anticipatory responses. The results showed differential effects of temporal stimulus uncertainty in the hierarchy of responses as the learning course progressed.
\end{abstract}

Keywords: motor learning, open system, dynamic system, potential information

\section{Introduction}

The human motor learning process is a perception-motor system that can be characterized as open and dynamic. Therefore the system must interact with the environment. This interaction allows the system to increase its complexity by breaking down stability and searching for more organized states. ${ }^{1-3}$ In a thermodynamic system, to break down stability and to keep itself far from equilibrium, negative entropy must be brought in. ${ }^{4}$ In a perception-motor system, information can be the negative entropy that the system needs to change toward a new state. As suggested by Stonier, ${ }^{5}$ information can be defined in terms of its capacity to organize a system, just as energy is defined in terms of its capacity to perform work.

Motor learning is then intimately related to the information that is available and interpretable in a performance instance. In agreement with Atlan, ${ }^{6}$ a system's quantity of information can be measured by its uncertainty. In fact, in accordance with Guadagnoli and Lee, ${ }^{7}$ information can be seen as a challenge to the motor skill learner for the following reasons: (a) learning cannot occur in the absence of information; (b) learning will be disrupted in the presence of too much or too little information; and (c) for learning to occur, there must be an optimal amount of information, which differs from the skill level of the individual and the difficulty of the to-be-learned task. Although Guadagnoli and $\mathrm{Lee}^{7}$ were aware that task difficulty has many definitions, it can be divided in two main categories. The first is nominal task difficulty, which is considered to reflect a constant amount of task difficulty, regardless of who is performing it and under what conditions it is being performed; this category includes such factors as perceptual and motor performance requests. The second category is functional task difficulty, which refers to how challenging a task is relative to the skill level of the

Correspondence: Maria Teresa Cattuzzo Rua Capitão Rebelinho, 720 ap.203, Pina, Recife (PE), Brazil CEP: 51011010

Tel +558 I 96930558

$\mathrm{Fax}+558131833354$

Email mtcattuzzo@hotmail.com 
individual performing it; that is, in the beginning of the motor learning process, there is more functional task difficulty than after a certain amount of practice. Finally, the uncertainty brought about by both task difficulties prompts the motor learning process.

To prepare for a situation that requires the organization of perceptual-motor aspects, the present study used a task that involved following a series of flashing lights. From the middle of the 20th century onwards, research has been directed toward understanding the mechanisms underlying skill acquisition in performing serial activities. ${ }^{8-14}$ The fluent production of serial movements requires the coordinated execution of skill components in a specific order and time, which typifies this task, such as following a choreographed dance.

It is important to consider that when subjects face a serial stimulus pattern for the first time, it does not actually constitute a serial task for them. It is, rather, a random one in the sense that the stimulus components are not linked to each other in a meaningful way. For the experimenter, however, it certainly is a serial task, but for the performers, the task will become serial as a function of practice. ${ }^{15}$ Practice in this context can be thought of as a problem-solving process that seeks to make order out of disorder, reducing the current system uncertainty in the task difficulty.

In particular, in the serial tracking tasks - ones in which the stimuli are presented in a repeated sequence - the subject may anticipate the upcoming stimulus, thus reducing the time required to respond. With additional practice, it is assumed that participants will be able to anticipate the upcoming stimulus, resulting in an even more rapid and fluid response production. ${ }^{16}$ Anticipatory responses in this context represent the most advanced level of performance of this task.

A fundamental assumption in the present study is that the motor learning process implies a qualitative change in performance by increasing the functional responses. Some studies ${ }^{17-20}$ have found that in the learning process of the serial tracking task, there is a hierarchy of responses. First of all, the system begins showing omission responses and then progresses to error, and finally, to correct and anticipatory responses. In other words, it is expected that information organizes the system, leading it to replace nonfunctional responses (ie, omission and error responses) with functional ones (correct and anticipatory responses).

By taking into consideration that the process of receiving information is critical to learning and that the temporal demand of the serial tracking must cause temporal uncertainty, but that practice can reduce the task difficulty, how the progressive increases in the temporal stimuli would be reflected on the system response hierarchy can be questioned. This study aims to investigate the effects of stimulus temporal demand through the manipulation of different inter stimuli intervals (ISI) on the emergence of omission, error, correct, and anticipatory responses during the acquisition of a serial tracking task by young men.

\section{Materials and method \\ Participants}

One hundred and twenty male adults ( 23 to 25 years old) were randomly distributed into six experimental groups composed of twenty subjects each, as shown in Table 1. The participants were volunteers - Police Formation Center (Pirituba, São Paulo, Brazil) students - who signed an informed consent statement before participating in the study, which was approved by the Physical Education and Sport School Ethics Committee, University of São Paulo.

\section{Apparatus and task}

A serial pattern learning apparatus was used; consisting of a stimulus display board composed of six light-emitting diodes arranged in a line with a fixed distance of $5 \mathrm{~cm}$ between them. Below the light-emitting diodes was a response keyboard with six buttons arranged in a line. The apparatus was connected to a notebook (Figure 1) installed with software designed to record the stimulus delivery and response. Both the apparatus and the software were developed in the Motor Behavior Laboratory of School of Physical Education and Sport of University of São Paulo, Brazil.

The task consisted of pressing the six buttons corresponding to a flashing sequence of the light-emitting diodes, which had a defined ISI: the timing interval between each of the six consecutive light signals in the sequence. The ISI values that characterized the six experimental groups were as follows: G1: ISI $=300 \mathrm{~ms}$; G2: ISI $=400 \mathrm{~ms}$; G3: ISI $=500 \mathrm{~ms}$; G4: ISI $=600 \mathrm{~ms}$; G5: ISI $=700 \mathrm{~ms}$; and G6: ISI $=800 \mathrm{~ms}$. The number of lights in the sequence (six) and the number of trials were defined by a pilot study that demonstrated that these ISI were difficult, but possible to learn in 100 trials.

Table I Mean and standard deviation of the performers' age in the six experimental groups

\begin{tabular}{lllllll}
\hline Groups & GI & G2 & G3 & G4 & G5 & G6 \\
\hline ISI (ms) & 300 & 400 & 500 & 600 & 700 & 800 \\
Age & & & & & & \\
Mean (years) & 23.9 & 23.7 & 24.2 & 25.0 & 23.8 & 24.6 \\
SD (years) & 3.0 & 2.6 & 2.6 & 3.9 & 3.2 & 3.3 \\
N & 20 & 20 & 20 & 20 & 20 & 20 \\
\hline
\end{tabular}

Abbreviations: ISI, inter stimuli intervals; SD, standard deviation; $N$, number. 


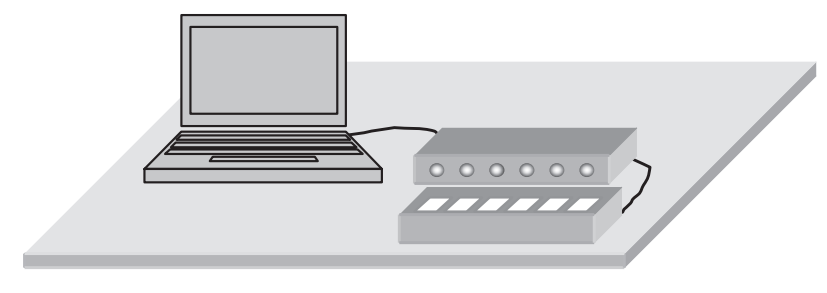

Figure I Experimental apparatus.

Based on work by Restle, ${ }^{21}$ the serial pattern adopted was 3-1-4-6-2-5, which avoided "easy-to-learn" patterns such as runs (1-2-3), trills (1-3-1), or repetitions (1-1-1). The referred sequence was the same in all different ISI values.

\section{Procedures}

The experiment was conducted in a quiet place, at room temperature, and under artificial and natural lights. Each performer was seated comfortably in a chair in front of a table where the apparatus was placed. All participants performed the task using their dominant hand. Verbal instructions about the use of the apparatus and the goal of the task were provide before the test. Subjects were required to respond as quickly and accurately as possible to the stimulus, and were instructed to try to identify the repeating sequence by pressing the buttons. Before the test, the performers each received five attempts to try out the apparatus, and then they performed 100 trials without interruption.

\section{Measures}

The dependent variables of the present study were as follows: number of missed responses, incorrect responses, correct responses, and anticipated responses. A missed response occurred when no response was detected between two sequential stimuli; an incorrect response occurred when the response did not correspond to the stimulus; a correct response occurred when the response corresponded to the stimulus; and an anticipatory response occurred when the correct response was made just before the presentation of a correspondent stimulus.

\section{Statistical procedures}

For the descriptive and inferential statistics, the proportion of responses was calculated for each block of ten trials, as shown in Figure 2. Multivariate analysis of variance and the Ryan-Einot-Gabriel-Welsch multiple range post hoc tests from the SAS Software 9.1 version $^{22}$ were used. The significance level was $P<0.05$. For the repeated measures, multivariate analysis of variance test was used for each of the three blocks of practice: B1 corresponds to the initiation of practice, $\mathrm{B} 5$ corresponds to $50 \%$ of practice, and $\mathrm{B} 10$ corresponds to the last block of practice.

\section{Results and discussion}

The aim of this study was to verify the effects of different stimulus temporal demands obtained by the manipulation of inter stimuli intervals during the acquisition of a serial tracking task. The planning, execution, and monitoring of movement sequences involve the control of the cognitive process, which the configuration of the movements reflects. The fluent production of sequential movements, such as those involved in the production of Morse codes, ${ }^{10}$ typing, ${ }^{11}$ piano playing,,${ }^{12,13}$ and guitar playing, ${ }^{14}$ have been the object of scientific inquiry for a number of applied and theoretical reasons. ${ }^{16}$

Figure 2 shows the results of the six experimental groups. It was expected that the perception motor system would replace the nonfunctional responses (ie, omission and error responses) with functional ones (correct and anticipatory responses) during the learning process. In fact, this occurred in all groups. Specifically, for groups G1 and G2, the missed responses and the incorrect responses, that is, the nonfunctional responses, constituted the largest proportion of the responses throughout the practice. In G1, more than 75\% of the responses were nonfunctional in most trial blocks. On the other hand, Figure 2 shows that for G3, the proportion of nonfunctional responses was about $50 \%$ in most blocks of trials, and this was the first group to show a gradual increase in anticipatory responses.

The graphs show that the smallest proportion of nonfunctional responses occurred in G4, G5, and G6, and the proportion of those responses seems to remain unchanged throughout the ten blocks of practice in G4 and G6, but not in G5. In addition, there were higher proportions of functional responses in groups with longer ISI (G4, G5, and G6). When specifically observing the anticipatory responses - defined as the highest response level - a gradual increase was shown during practice only for G3, G5, and G6.

Inferential analysis confirmed that ISI affected the global performance of all groups. There was interaction effect on the anticipatory $(\mathrm{F}=3.85 ; P=0.0001)$, correct $(\mathrm{F}=8.17$; $P<0.0001)$, incorrect $(\mathrm{F}=3.70, P<0.0001)$, and omission responses $(\mathrm{F}=2.46 ; P<0.0081)$. This means that the temporal uncertainty affected the qualitative order of responses during practice.

In this study, the stimulus temporal uncertainty specified by means of ISI was understood as a nominal difficulty. ${ }^{7}$ As such, it generates uncertainty in the system and potential information for learning, but practice appears to be an order 
G1-300 ms

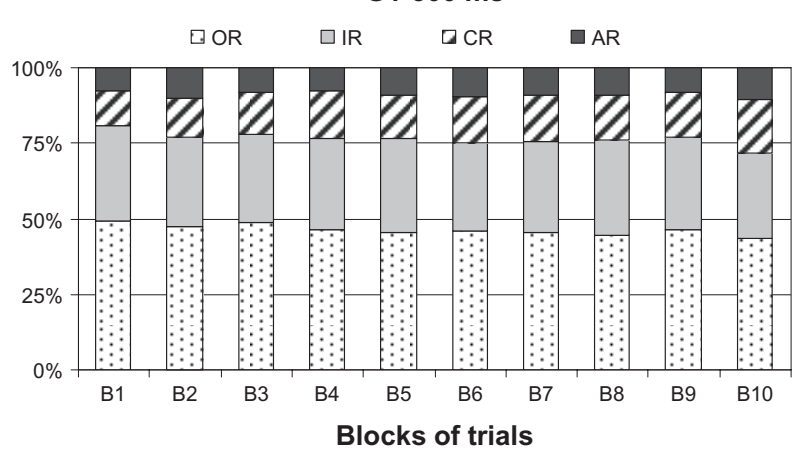

G3-500 ms

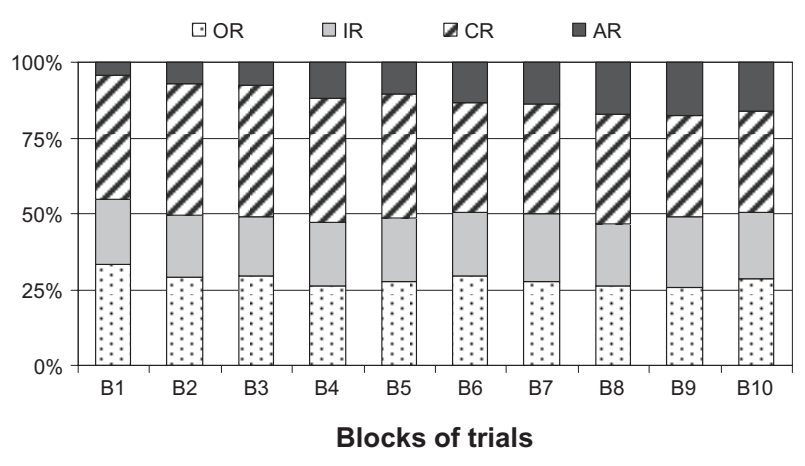

G5-700 ms

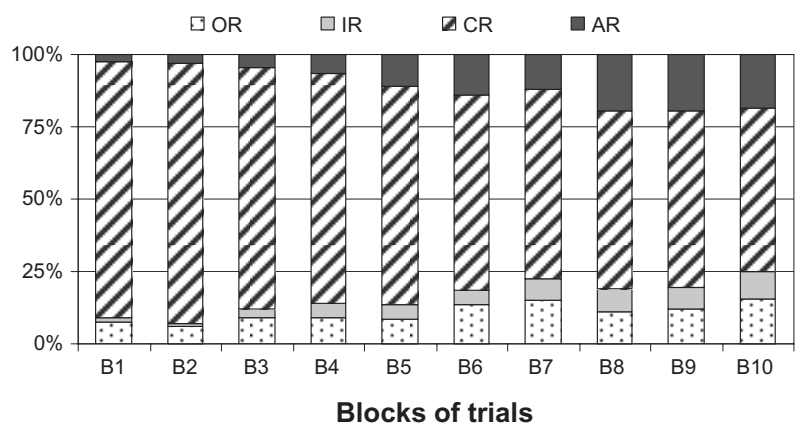

G2-400 ms

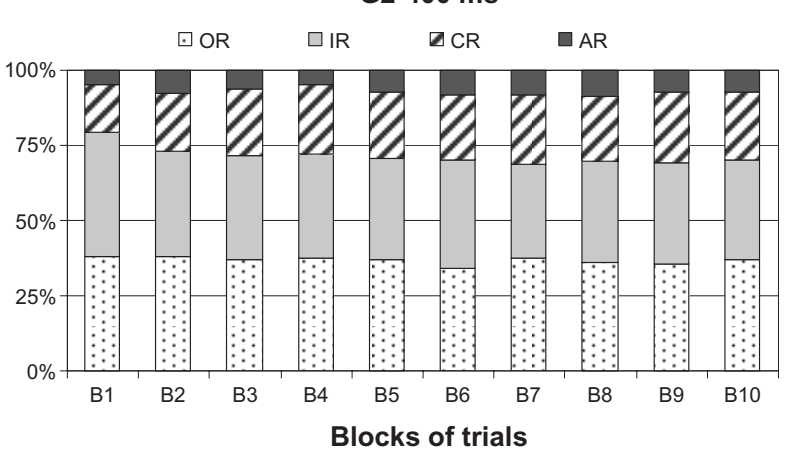

G4-600 ms

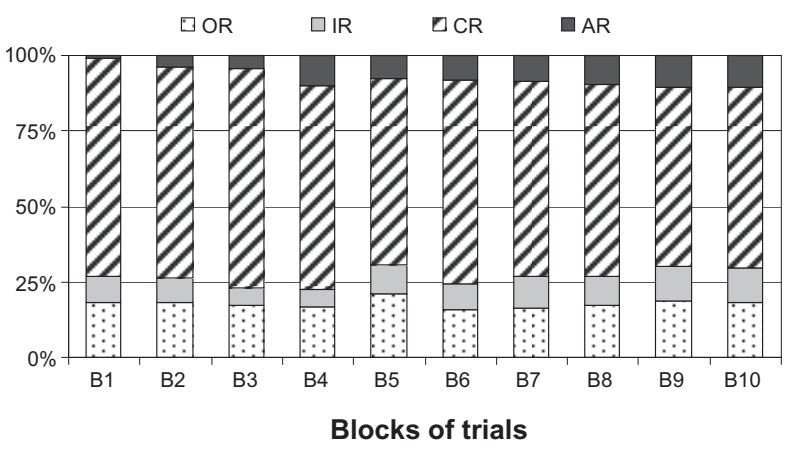

G6-800 ms

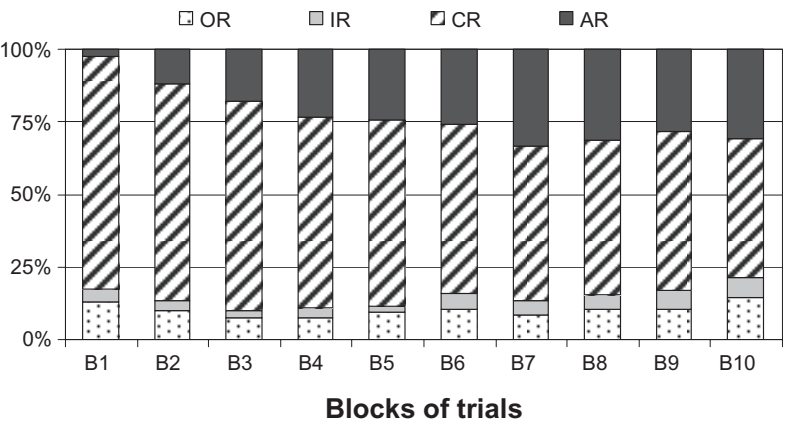

Figure 2 Proportion of omission (OR), incorrect (IR), correct (CR), and anticipatory (AR) responses by blocks of trials for the six experimental groups (GI, G2, G3, G4, G5, and G6).

factor for the system because it reduces uncertainty. The responses in this study must reflect a hierarchical order of responses in the serial tracking pattern learning process. ${ }^{16}$ It was expected that in the global performance evaluation, functional responses would replace nonfunctional ones during the learning process and that when a complete serial pattern was performed with anticipatory responses, one might assume that an order parameter had emerged from a dynamic interaction of components, which in turn controls their behavior.

G1 and G6 were submitted to extreme ISI (the shortest and the longest, respectively). A comparison of these groups' performances represents how the stimulus temporal demand affected learning. In the initial block of practice, G1 showed the highest mean value for anticipatory responses, which differed statistically from G6's mean value. After completion of $50 \%$ of the experiment, both groups continued to improve their performance, but at this time, G6 presented the highest mean value for anticipatory responses, which, however, were not statistically different from the mean value of G1. In the last part of the experiment, both groups continued to improve their performance for anticipatory responses, but G6 more than doubled the number of anticipatory responses of G1, and this result was statistically significant. An explanation for the initial performance of G1 regarding these anticipatory responses would be that the interval adopted for it (ISI $=300 \mathrm{~ms}$ ) would allow only the perception motor system to react to the luminous stimulus 
individually. This means that the sequence of lights had not been understood as a pattern, that is, the nominal difficulty was very high. Thus, the anticipatory responses can be an order parameter only for G6.

With regard to the correct responses, both groups improved their performance throughout the practice. In the early and middle parts of the practice, the mean values of G6 were statistically higher than those of G1, but in the last part, the difference between the mean values of the two groups was not significant. The gradual, functional response performance improvement by both groups and the absence of a significant difference between them in the production of correct responses at the end of the practice suggest that even though G1 had a higher demand of information processing than G6, such a demand was liable to be met and used by the system. To this end, G1 required additional practice.

Regarding error and omission responses produced by all groups, G1 presented the highest level of error responses and the second highest level of omission responses throughout the practice. G6 showed performance variation during practice; first, its level of performance concerning omission and error responses were lower from $\mathrm{B} 1$ to $\mathrm{B} 5$, but then increased in the last block of practice. The $800 \mathrm{~ms}$ (ISI) G6 interval seems to imply a low processing demand, allowing the system to explore other responses, which, however, elevated the number of nonfunctional responses.

In this study, in the beginning of the learning process of the serial tracking task, the subjects seem to be susceptible to the time limits imposed on information processing, or the named nominal difficulty: the shorter the ISI is, the fewer the functional responses are. Throughout the practices, however, these time limits lost their importance because the stimulus pattern regularity was perceived and the performers, even the ones from the shortest ISI groups, began presenting more functional responses (correct and anticipatory responses). Nevertheless, a proportion of these responses were affected by ISI.

These results suggest that performance depends on the resources the system uses to overcome the uncertainty that is associated with the information processing demands of all ISI. Nevertheless, practice, a process that instills order to the system, allowed the search of more organized states, ie, functional responses.

Another point of emphasis is that a lower level of temporal uncertainty does not ensure a better performance, as already established in a study by Meira Junior. ${ }^{23}$ In the present study, G6 performance may serve as an example of this statement since it was the group with the longest ISI, and consequently, with the lowest level of uncertainty or nominal difficulty. As to the functional responses, their anticipatory mean values did not differ from those of the groups with lower ISI, such as G3 (ISI $=500 \mathrm{~ms}$ ) and G5 (ISI = $700 \mathrm{~ms}$ ). Regarding the correct responses, G6 behaved similarly to all groups in the latter parts of the practice. In addition, considering the nonfunctional responses, G6 behaved identically to groups G4 and G5, which showed a high level of stimulus temporary uncertainty with shorter ISI.

Finally, the interval assigned to G3 generated a degree of uncertainty that challenged the system to go on searching for the best response to meet the requirement of learning the sequence. Functional and nonfunctional responses were produced in equal proportions at the beginning of the practice; however, in the latter part, there was an increase in the proportion of functional responses.

\section{Conclusion}

Comparative group analyses demonstrated that a difference in ISI led to different levels of requirement for the learning process, and that practice, an order generating factor, influenced subjects' performance. To sum up, the results of this study permit some reflection regarding the role of potential information for performance and learning. The ISI in the groups G1 and G2 were considered to cause high nominal difficulty and low potential information because these groups presented a high level of nonfunctional responses throughout the practice. In contrast to this, the ISI of groups G4, G5, and G6 were considered to cause a low level of nominal difficulty and low potential information because they permitted the system to present a high level of functional responses throughout the practice. The ISI of G3 (500 ms) apparently provided satisfactory potential information to the system since the group increased the functional responses during practice.

In conclusion, the changes in functional response patterns in this study seem to reflect a hierarchical order of responses in the learning process of serial tracking patterns.

\section{Acknowledgments}

The study was carried out with financial support from the Coordination of Improvement of Higher Education Personnel - Ministry of Education (CAPES - project PQI-0114030). The participants are gratefully acknowledged, and sincere thanks are given to Letícia Silva do Nascimento for all the support given during this work.

\section{Disclosure}

The authors report no conflicts of interest in this work. 


\section{References}

1. Bertalanffy L Von. General System Theory: Foundations, Development, Applications. New York: George Braziller; 1968.

2. Prigogine I, Stengers I. The new alliance: metamorphosis of science. Brasília: Ed. UnB; 1984. [in Portuguese]

3. Schneider ED, Kay JJ. The order from disorder: the thermodynamics of biological complexity. In: Murphy MP, O’Neill LA, Editors, What is life? 50 years later. Speculation about the future of biology. São Paulo: Foundation UNESP Publisher, 1997. [in Portuguese]

4. Schrödinger E. What is Life? Cambridge: Cambridge University Press; 1944.

5. Stonier T. Information as a basic property of the universe. Biosystems. 1996;38:2-3.

6. Atlan H. Entre el cristal y el humo: ensayo sobre la organización de lo vivo. (Between the glass and smoke: an essay on the organization of the living). Madrid: Debate; 1990. [in Spanish]

7. Guadagnoli MA, Lee TD. Challenge point: a framework for conceptualizing the effects of various practice conditions in motor learning. J Mot Behav. 2004;36(2):221-224.

8. Lashley KS. The problem of serial order in behavior. In: Jeffress LA, editor. Cerebral Mechanisms in Behavior. New York: Wiley; 1951: $112-136$.

9. Shin JC, Ivry RB. Concurrent learning of temporal and spatial sequences. J Exp Psychol Learn. 2002;28(3):445-457.

10. Klapp ST. Motor response programming during simple and choice reaction time: the role of practice. J Exp Psychol Human. 1995;21(5):1015-1027.

11. Rumelhart DE, Norman DA. Simulating a skilled typist: a study of skilled cognitive-motor performance. Cogn Sci. 1982;6:1-36.

12. Repp BH. Detecting deviations from metronomic timing in music: effects of perceptual structure on the mental timekeeper. Percept Psychophys. 1999;61(3):529-548.

13. Shaffer LH, Clarke E, Todd NP. Meter and rhythm in piano playing. Cognition. 1985;20(1):61-77.
14. Heijink H, Ruud G, Meulenbroek J. On the complexity of classical guitar playing: functional adaptations to task constraints. JMotor Behav. 2002;34(4):339-351.

15. Tani G. Hierarchical organization of an action program and the development of skilled actions. Unpublished Technical Report. Department of Psychology of University of Sheffield, Sheffield; 1995.

16. Park JH, Shea CH. Sequence learning: response structure and effector transfer. Q J Exp Psychol A. 2005;58A(3):387-419.

17. Choshi K. Chikaku undo gakushu ni okeru hanno no jikanteki kozo (Temporal structure of response in perceptual-motor skill learning). Memoirs of the Faculty of Integrated Arts and Sciences. 1983:29-38. [in Japanese].

18. Choshi K. Chikaku undo gakushu no tekiyo katei - fukuzatsusei to sohosei (Adaptive process of perceptual-motor learning: complexity and complementarity). Memoirs of the Faculty of Integrated Arts and Sciences. 1986;3:21-30. [in Japanese].

19. Choshi K, Tani G. Stable system and adaptive system in motor learning [in Japanese]. In: Japanese Association of Biomechanics, editor. The Ccience of Movement $V$. Tokyo: Kyorin, 1983.

20. Cattuzzo MT. The instability-stability-instability cycle in the adaptive process of motor learning. Doctoral Thesis. São Paulo: Escola de Educação Física e Esporte; 2007. http://www.teses.usp.br/teses/ disponiveis/39/39132/tde-24042008-064211/.

21. Restle F. Theory of serial patterns learning: structural trees. Psychol Rev. 1970;77(6):481-495.

22. SAS/STAT. Software Changes and Enhancement. Cary, NC: SAS Institute North Caroline; 1997.

23. Meira Júnior CM. Knowledge of results on adaptive process in motor learning. Doctoral Thesis. São Paulo: Escola de Educação Física e Esporte; 2005. http://www.teses.usp.br/teses/disponiveis/39/39132/ tde-17082006-075844/.
Psychology Research and Behavior Management

\section{Publish your work in this journal}

Psychology Research and Behavior Management is an international, peerreviewed, open access journal focusing on the science of psychology and its application in behavior management to develop improved outcomes in the clinical, educational, sports and business arenas. Specific topics covered include: Neuroscience, memory \& decision making; Behavior

\section{Dovepress}

modification \& management; Clinical applications; Business \& sports performance management; Social and developmental studies; Animal studies. The manuscript management system is completely online and includes a quick and fair peer-review system. Visit http://www.dovepress. com/testimonials.php to read real quotes from published authors. 